\title{
Quantifying Cognitive State From EEG Using Dependence Measures
}

\author{
Bilal Fadlallah*, Member, IEEE, Sohan Seth, Member, IEEE, Andreas Keil, and José Príncipe, Fellow, IEEE
}

\begin{abstract}
The exquisite human ability to perceive facial features has been explained by the activity of neurons particularly responsive to faces, found in the fusiform gyrus and the anterior part of the superior temporal sulcus. This study hypothesizes and demonstrates that it is possible to automatically discriminate face processing from processing of a simple control stimulus based on processed EEGs in an online fashion with high temporal resolution using measures of statistical dependence applied on steady-state visual evoked potentials. Correlation, mutual information, and a novel measure of association, referred to as generalized measure of association (GMA), were applied on filtered current source density data. Dependences between channel locations were assessed for two separate conditions elicited by distinct pictures (a face and a Gabor grating) flickering at a rate of $17.5 \mathrm{~Hz}$. Filter settings were chosen to minimize the distortion produced by bandpassing parameters on dependence estimation. Statistical analysis was performed for automated stimulus classification using the Kolmogorov-Smirnov test. Results show active regions in the occipito-parietal part of the brain for both conditions with a greater dependence between occipital and inferotemporal sites for the face stimulus. GMA achieved a higher performance in discriminating the two conditions. Because no additional face-like stimuli were examined, this study established a basic difference between one particular face and one nonface stimulus. Future work may use additional stimuli and experimental manipulations to determine the specificity of the current connectivity results.
\end{abstract}

Index Terms-Brain connectivity, correlation, electroencephalogram (EEG), finite impulse response (FIR) least-square filter, generalized measure of association (GMA), mutual information (MI), steady-state visual evoked potential (ssVEP).

\section{INTRODUCTION}

$\mathbf{T}$ HE human brain is one of the most complex self-organizing systems known. It roughly consists

Manuscript received February 20, 2012; revised May 23, 2012; accepted June 25, 2012. Date of publication July 25, 2012; date of current version September 14, 2012. This work was supported by the National Science Foundation under Grant IIS-0964197 and the Lebanese Center for Scientific Research. Asterisk indicates corresponding author.

${ }^{*} \mathrm{~B}$. Fadlallah is with the Department of Electrical and Computer Engineering and Computational NeuroEngineering Laboratory, University of Florida, Gainesville, FL 32611 USA (e-mail: bhf@cnel.ufl.edu).

S. Seth is with the Department of Information and Computer Science, Aalto University, FL-00076 Aalto, Finland, and also with the Helsinki Institute for Information Technology, FL-00014 Helsinki, Finland. (e-mail: sohan.seth@hiit.fi).

A. Keil is with the Department of Psychology and NIMH Center for the Study of Emotion and Attention, University of Florida, Gainesville, FL 32611 USA (e-mail: akeil@ufl.edu).

J. Príncipe is with the Department of Electrical and Computer Engineering and Computational NeuroEngineering Laboratory, University of Florida, Gainesville, FL 32611 USA. (e-mail: principe@ cnel.ufl.edu).

Color versions of one or more of the figures in this paper are available online at http://ieeexplore.ieee.org.

Digital Object Identifier 10.1109/TBME.2012.2210283 of 100 billion neurons, each having on average 7000 synaptic connections with its surrounding. The enormous structural connectivity of the brain complicates the complete understanding of its cognitive processes, which involve large networks in several cortices. However, there has been evidence that areas that are coactive during cognition can be as well interdependent, i.e., functionally connected, forming a cognitive control network [1], [2]. Based on this observation, it is only natural to explore the functional connectivity as a tool to quantify a cognitive state. In this paper, we study this particular aspect of cognitive neuroscience, with a strong focus on discriminating two cognitive states in statistical terms solely from the associated functional connectivity across brain regions. To elaborate, we study the difference in activated regions when the visual stimulus is either a neutral facial stimulus (Face) or a contrast-matched control stimulus (Gabor patch).

Previous studies of social perception have indicated that features bearing motivational relevance undergo facilitated visual cortical processing [3], [4]. The same applies to stimuli with higher object-based complexity or specific semantic content. In spite of the fact that the neural mechanisms controlling this facilitation remain unknown, much of the present evidence seems to point toward the amygdaloid complex and the parietofrontal cortex as origins of reentrant modulation into lower tier visual areas, when perceiving biologically significant stimuli [5], [6]. According to this hypothesis, visual perception and attention to relevant stimuli involve communication between the occipital and frontal cortices mediated by subcortical structures [7], [8]. Todorov and Engell [9] have also suggested that novel faces are automatically evaluated by the amygdala according to a general valence dimension causing the activation of a face responsive network in the occipital and temporal cortices, whereas the contribution of the fusiform gyrus and superior temporal sulcus in processing facial structures has been studied in [10] and [11] and others. Therefore, we exploit the fact that an increased functional connectivity in and among these areas may represent a potential index of the presence of facial visual stimulation, compared to a standard visual pattern, lacking biological significance.

The activity in the relevant areas can be observed and recorded by a number of noninvasive methods such as MRI, functional MRI (fMRI), EEG, and magnetoencephalography (MEG). MRI and fMRI have the advantage of providing an excellent spatial resolution, but this comes at the expense of a poor time resolution, caused by their dependence on blood flow. Given that neural communication ranges in time scales between 1 and 100 $\mathrm{ms}$ depending on the neurons characteristics [12], fMRI is not well suited to assess cognitive tasks where time scales of interest do not exceed $100 \mathrm{~ms}$. On the other side, the high temporal 
resolution of EEG and MEG $(\sim 1 \mathrm{~ms})$ make them a better tool to study the dynamics of brain activity. However, EEG recordings are inherently noisy, and therefore, we here rely on the steadystate visual evoked potential (ssVEP). ssVEPs are continuous brain responses caused by repetitive visual stimuli, generally modulated in intensity with a fixed rate usually less than $30 \mathrm{~Hz}$ and not smaller than $3 \mathrm{~Hz}$ [13]. These scalp potentials can be captured as signals oscillating with a fundamental frequency equal to the stimuli flashing rate. ssVEPs have recently become a popular tool in cognitive and clinical neuroscience [3], [14]-[17]. They are easy to induce and have several other advantages like good signal-to-noise ratio (SNR) and relative immunity to artifacts. In addition, they require minimal subject training, and simplify feature extraction and analysis in frequency space.

Given the complexity of cortical activity across recording sites, one important step is to quantify the functional connectivity among scalp locations and, ultimately, brain areas. This can be done in several ways, which are usually categorized as time-domain [3], [18], frequency-domain [19]-[22] and timefrequency methods [23], [24]. In this paper, we consider the first approach since it is fairly possible that a cognitive state is highly volatile, and therefore, it should be quantified only over a short period of time, i.e., with high time resolution. This can be done preferably in the time domain, since frequency-domain approaches sacrifice the time resolution in order to extract precise frequency information. Often electrical activity is measured over time across several cortical areas, and the standard practice quantifies the association of the signals in terms of correlation [25]-[28] or mutual information (MI) [29]-[31]. However, both approaches have certain drawbacks. For example, correlation is a measure of linear dependence, and it is perhaps a strong assumption that two brain regions communicate through a linear channel. On the other hand, although MI provides a more rigorous quantification of association between two signals, it is rather difficult to estimate; especially when the sample size is small and dimensionality is high, which is pertinent to our objectives of preserving time resolution and assessing dependence over considerable propagation delay. Moreover, the estimation of MI requires selecting a free parameter, which adds more uncertainty to the analysis. To tackle these problems, we use a generalized measure of association (GMA) as a measure of dependence. Typical advantages of GMA are that it is parameter-free, and that it provides an intuitive understanding of dependence in the context of realizations. These are important when we only have a few samples - a situation where traditional estimators become highly biased, thus losing their meaning. We compare the performance of GMA against correlation and MI, and show that it indeed better quantifies the functional connectivity.

The rest of this paper is organized as follows. In Section II, we describe the experimental setting and outline the initial preprocessing performed to extract current source densities (CSDs) from scalp potentials. Section III describes the signal processing approach and in Section IV, we analyze the frequency components of the obtained signal, processed according to a robust filtering scheme that reduces the sensitivity of the dependence backend to bandpassing parameters. In Section V, we examine two approaches in the literature that can be used to analyze de- pendence between different electrode measurements and infer associations between the corresponding brain locations. Besides these measures, namely correlation and MI, we also describe GMA as a novel rank-based measure of dependence. Section VI provides an overview of results from the previously mentioned perspectives based on a standard formulation of the problem, and Section VII offers discussion and concluding remarks.

\section{MATERIALS AND MEthodS}

\section{A. Stimuli and Recordings}

Overview: The experiment included two participants. Both were male graduate students in their early twenties who gave written consent prior to taking part in the study. The subjects had normal vision and no family history of epilepsy and did not report any psychotherapeutic history. The procedure was approved by the institutional review board of the University of Florida. Because of the reduced number of subjects, the primary goal of this paper is to concentrate on the signal processing aspects of the project and validate the algorithms with real human data originating from more than just one individual. A group study will be the object of future work.

Procedure: After application of the electrode net of a 129channel HydroCell Geodesic Sensor Net montage [32], and setting electrode impedances below $50 \mathrm{k} \Omega$ according to the manufacturer's recommendation for the high-impedance amplifiers, the experimental session proceeded with continuous recordings using $C_{z}$ as recording reference. Epochs of $400 \mathrm{~ms}$ prior to stimulus onset and $4200 \mathrm{~ms}$ after onset were extracted for a total of 4.6 s recordings. Subsequent to the baseline segment, an image showing a neutral human face was presented to the subject on a $17^{\prime \prime}$ monitor with a vertical refresh rate of $70 \mathrm{~Hz}(17.286 \mathrm{~ms}$ per screen duty cycle). Faces were shown for two duty cycles of the display and then turned off for two cycles, leading to on-off (square-wave) flicker at $17.5 \mathrm{~Hz}$. This corresponds to 73 presentations of each stimulus per trial.

The same procedure was repeated with a control stimulus showing a Gabor patch, i.e., a pattern of stripes, where patches are calculated on the fly using pixels of the face picture. Luminance for both pictures was set to vary from near-zero to $52 \mathrm{~cd} \cdot \mathrm{m}^{-2}$ and both were matched for mean luminance (i.e., $9.7 \mathrm{~cd} \cdot \mathrm{m}^{-2}$ ), average contrast (i.e., $50 \%$ ), and mean spatial frequency (i.e., $4 \mathrm{cpd}$ ) to preclude systematic differences with respect to these parameters. With these settings, stimuli are well below the flicker fusion frequency, and stable ssVEP amplitude can be expected [33].

Gabor patches have been extensively used as control stimuli in a variety of contexts [34]-[36], and the aim of this study was to examine the discriminability between a face and a nocontent pattern. Thus, in this paper, the two stimuli are considered from the perspective of inferring discriminating features without particularly stressing the characteristics of the specific physiological processes associated with face processing. All stimuli flickered at a frequency $F_{o}=17.5 \mathrm{~Hz}$ and a total of $N_{t}=15$ trials were performed for each stimulus condition (this is deemed sufficient to yield a stable response for any subject and condition). From this point onward, we refer to the facial 
stimulus as "Face" and the Gabor stimulus as "Gabor patch." During the experiment, the subject was asked to attentively maintain gaze on the pictures, not to blink, and avoid as much as possible movements of the eye and the head. EEG data were collected from the recordings of the $N_{c}=129$ electrodes, at a digitization rate of $F_{s}=1000 \mathrm{~Hz}$. In a typical scenario, we would perform offline artifact rejection (that might include some visual inspection steps) to detect individual channel artifacts and interpolate potentials at these channels via spherical splines. This was not performed in our case since it was not clear how it would affect computed dependences in sensor space.

\section{B. Volume Conduction and CSDs}

Several factors interfere with the process of getting a highquality representation of brain activity when recording an EEG signal. The impact of some of these artifacts can be attenuated like the subject's head motion and eye blinking, whereas others are uncontrollable such as the electrical activity of some muscles, electrocardiograms, and especially the effect of volume conduction. To reduce the effect of the latter, we estimate the strength of extracellular current generators underlying the recorded scalp potentials. This is done using a spherical model of the head based on a linear volume conduction assumption. Such model includes four layers (scalp, skull, cerebrospinal fluid, and brain) and is convenient since it allows derivation of analytic results with low margin of error [37]. As a result, we use CSD measures that roughly approximate the locations of the current sources and sinks in a reference-free fashion [38], [39]. To derive these measures, we follow the procedure outlined in [40] where the calculation of the CSD or the equivalent "Laplacian" method is useful to reduce the spatial low-pass filtering impact caused by the volume conduction property of the tissue, fluids, and skull [41], [42]. This method primarily relies on a sufficient spatial sampling as well as an adequate SNR [40]. These requirements were met for this study with event-related potential measurements performed at 129 electrode locations with 15 trials per condition.

\section{Data}

The recording at each channel location $i$ was collected as a time series $x_{i}^{(k)}$ for face and $y_{i}^{(k)}$ for Gabor path per trial $k$. As a result, the whole data can be represented as two spatiotemporal data matrices $\mathbf{X}^{(k)}=\left[x_{1}^{(k)}, \ldots, x_{N_{c}}^{(k)}\right]^{T}$ and $\mathbf{Y}^{(k)}=\left[y_{1}^{(k)}, \ldots, y_{N_{c}}^{(k)}\right]^{T} \in \mathbb{R}^{N_{c} \times N_{s}}$, where $N_{c}$ and $N_{s}$ denote the number of channels and sampled data points per recording, respectively. $N_{c}=129$ and for $F_{s}=1000$ and a downsampling factor $d=1, N_{s}=4600$. For later use, we also define $N_{r}=400$ to be the number of samples for the baseline segment and for a given vector $a$, the notation $a\{n, m\}$ denotes the subvector $\left\{a_{n}, \ldots, a_{m}\right\}$.

\section{SignAl PROCESSING}

The recorded EEG signals reveal the presence of strong noises at $60 \mathrm{~Hz}$ and its odd harmonics (third and fifth), which suggests the use of notch filters to preprocess the data prior to extracting

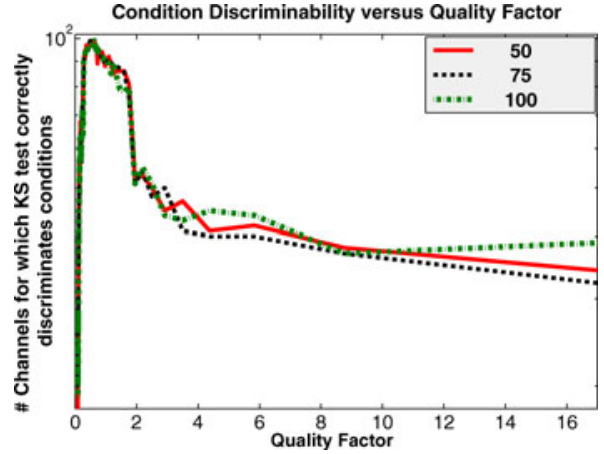

Fig. 1. Plot showing the change in the number of channels for which the two conditions are correctly discriminated by the KS test as the bandpass filter quality factor increases. Simulations were performed for three filter orders and GMA was used as dependence measure. The curve increases with the quality factor, reaches a plateau, and then decreases as the quality factor goes up, clearly showing a region where the estimated dependence values are robust.

the frequency band of interest. It is known that filters with higher quality factors induce better stopband attenuation but exhibit more ringing. For functional dependence studies, it is desirable to design a filter that can capture all the ssVEP relevant modulation while preventing ringing as much as possible since this may provide spurious dependences across channels that are independent. The impact of the filter's order and quality factor has been thoroughly assessed in [43] where the evaluation criterion of the filtering scheme was chosen to be the discriminability between the two conditions in response to variations in these two parameters. The considered measure of dependence was GMA, which will be described in detail in Section VI. Details about the filters used to process the recorded signals are included in [43] and it has been shown that the best set of parameters in terms of reducing the sensitivity of GMA values to the bandpassing scheme and achieving a better separation between the two conditions consists of a quality factor approaching unity and a filter order in the range $[50,100]$. This is illustrated in Fig. 1. In this paper, an order-70 filter with unity quality factor is used.

\section{FREQUENCY-DOMAIN ANALYSIS}

We first analyze the filtered signals in the frequency domain over the sensor space to assess the filtering impact and localize the power in the $F_{o}$ range. Power is visualized for different frequency bands between 16 and $18.9 \mathrm{~Hz}$. For both conditions, power in a $0.5-\mathrm{Hz}$ band including $F_{o}$ clearly dominates those in the other ranges and is mostly concentrated in the right occipitoparietal regions. This is shown in Fig. 2 where power was averaged over all trials and plotted by sensor location.

The variability per trials of the fast Fourier transform (FFT) power at the flickering frequency is shown in Fig. 3. The computed variance per trial is inline with the power distribution plot (see Fig 2, upper row) that shows more localization of the power at $17.5 \mathrm{~Hz}$ for the face condition. Fig 3(b) illustrates two different linear fits between the variance vectors quantiles with standard normal quantiles for the two conditions.

Although the basic power spectral density approach seems to distinguish the two conditions, the statistical significance of the 


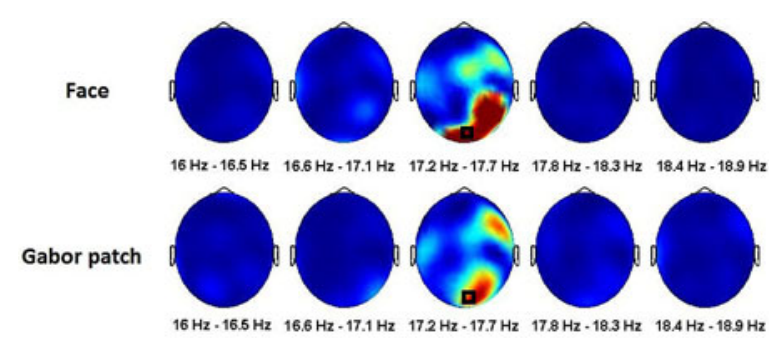

Fig. 2. FFT power in electrode space for filtered signals averaged over trials. Upper row corresponds to the face condition and lower row corresponds to the Gabor patch condition. Both conditions show distinctively high power in the flickering frequency band mostly concentrated in the right occipito-parietal region determined by $\left(\mathrm{O}_{2}, P_{z}, P_{4}\right)$. The location of channel 72 or $\mathrm{PO}_{z}$ is indicated by the black square.

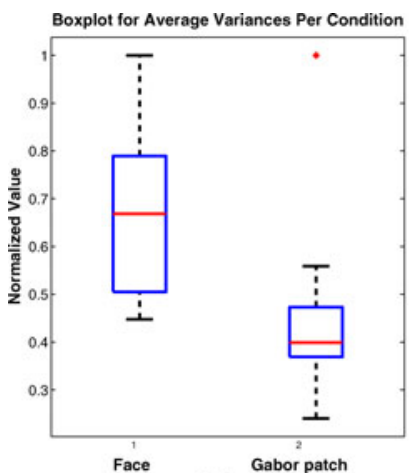

(a)

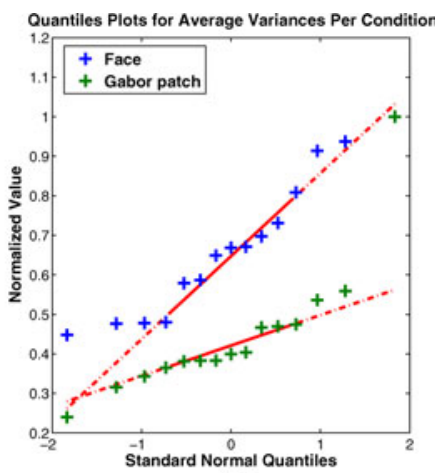

(b)
Fig. 3. Assessing power variability per trial at the flickering peak. (a) Boxplot for vectors representing the variance of the power at $F_{o}$ per condition across all trials. Edges of the box correspond to the 25th and 75th percentile. (b) Plot of each vector's quantiles with respect to standard normal quantiles illustrates the difference in distributions between the two conditions.

differences between the two conditions is questionable. Even if we assume otherwise, the time resolution provided via this approach is very limited. Thus, in order to quantify the dependence between electrode locations in response to the visual stimuli, we apply dependence measures on time windows of the filtered signals. Such approach bypasses the need to extract time delays from the signal's phase. Besides, it performs better than a classifier based only on power discrimination (as illustrated in Fig. 3). We hence tackle the problem from a time-domain perspective, using directly the time series processed by the linear-phase finite impulse response filter previously discussed to provide a better discrimination between the two conditions.

\section{DePendence Measures in the Time Domain}

Dependences are computed per trial for every pair of channels. As a preliminary test for this methodology, we select a channel location in the occipito-parietal region, corresponding to location $P O_{z}$ in an extended 10-20 system, to display a representative map of the obtained dependences. Since we know that ssVEPs originate mostly from visual cortical areas [44], we provide the following motivation facts to support this choice: 1) the proximity of this location to the occipital regions where the signal is known to originate from (as shown in Fig. 2) and 2) the unbiasedness of this location toward either of the right or left brain hemispheres. The advantage sought by selecting this channel consists in providing an insight about how the occipital region of the brain relates in time to the surrounding occipito-temporal and parietal areas. Hence, for each dependence measure and condition, an $N_{c} \times N_{t} \times N_{w}$ matrix was computed where $N_{w}$ denotes the number of time windows over which dependence is computed. For $d=1$ and a time window length of $114 \mathrm{~ms}, N_{w}=36$.

\section{A. Pearson's Product-Moment Coefficient}

A correlation measure between two variables reflects the degree to which the variables are related. The most widely used measure of correlation is the Pearson productmoment correlation defined for two variables $Q$ and $R$ as

$$
\rho(Q, R)=\frac{\operatorname{cov}(Q, R)}{\sigma_{Q} \sigma_{R}}=\frac{E\left[\left(Q-\mu_{Q}\right)\left(R-\mu_{R}\right)\right]}{\sigma_{Q} \sigma_{R}}
$$

where $\sigma$ serves to denote standard deviation and $\mu$ is the mean. The aforementioned quantity can be estimated for a sample of size $n$ using statistical estimators of the variance and covariance

$$
r(Q, R)=\frac{\sum_{i=1}^{n}\left(Q_{i}-\bar{Q}\right)\left(R_{i}-\bar{R}\right)}{\sqrt{\sum_{i=1}^{n}\left(Q_{i}-Q\right)^{2}} \sqrt{\sum_{i=1}^{n}\left(R_{i}-\bar{R}\right)^{2}}}
$$

where $\bar{Q}$ denotes the average of $Q$. Pearson's correlation reflects the strength of the linear relationship between two variables. It ranges from +1 to -1 corresponding, respectively, to reflect positive and negative linear relationship between the two variables.

\section{B. $M I$}

Another popular approach to measure dependence between two time series is MI. Assuming $Q$ and $R$ have joint probability distribution $P_{Q R}(q, r)$, the MI between the two variables is defined as

$$
\begin{aligned}
I(Q, R) & =\sum_{q, r} P_{Q R}(q, r) \log \frac{P_{Q R}(q, r)}{P_{Q}(q) P_{R}(r)} \\
& =E_{P_{Q R}}\left[\log \frac{P_{Q R}}{P_{Q} P_{R}}\right]
\end{aligned}
$$

where $E_{P}$ represents the expected value operator over distribution $P$, and $P_{Q}(q)$ and $P_{R}(r)$ denote the marginal distributions: $P_{Q}(q)=\sum_{r} P_{Q R}(q, r)$ and $P_{R}(r)=\sum_{q} P_{Q R}(q, r)$. An alternative definition of MI using the definition of the entropy and conditional entropy quantities is $I(Q, R)=H(Q)-H(Q \mid R)$. MI can be then seen as the reduction in a variable's uncertainty when the second variable is known.

Estimating $\mathrm{MI}$ is a challenging problem. In this paper, we use the estimator proposed by Kraskov et al. [45]. Unlike conventional MI estimators based on binning or kernel density estimation, this method relies on entropy estimates using the $k$-nearest neighbor $(k-\mathrm{nn})$ algorithm. The expression of the estimator is shown as follows:

$$
\hat{I}(Q, R)=\psi(k) \varphi\left[\left(\psi\left(n_{q}+1\right)+\psi\left(n_{r}+1\right)\right)\right]+\psi(n)
$$


where $\varphi[]=.N^{-1} \sum_{i=1}^{N} E[.(i)], \psi(x)$ is the digamma function and $n_{e}(i)$ for a random variable $E$ represents the number of points $e_{j}$ whose distance from $e_{i}$ is strictly less than a certain distance $\epsilon_{i}$ computed as the difference between $z_{i}(Z$ denoting the maximum norm over the space consisting of the two random variables) and its $k$ th neighbor. In this paper, we use a value of $k=4$, after several trials.

\section{GMA}

Since correlation only captures second-order interactions, it performs poorly for time series where the random variables may be coupled in the high-order moments. Besides, setting the free parameter in an optimal way when estimating MI is not an easy task. We hence employ a novel rank-based measure of dependence capable of capturing nonlinear structure called GMA [46]. Recall that association between random variables $U$ and $V$ measures how much large values on $U$ are associated with large values on $V$. GMA generalizes the concept of association by considering the distance between realizations rather than their absolute locations, retains certain desired invariance properties of a measure of association, and only requires that they be defined in a metric space.

More specifically, a given set of realizations $\left\{u_{i}, v_{i}\right\}_{i=1}^{n}$ is defined to be dependent if pairs of close realizations of one random variable $\left(\left\{u_{i}, u_{j}\right\}\right)$ are associated with pairs of close realizations of the other random variable $\left(\left\{v_{i}, v_{j}\right\}\right)$. Here, closeness is defined in terms of the respective distance metrics of the spaces where the realizations lie in, i.e., $\mathcal{U}$ and $\mathcal{V}$. We, respectively, denote these distance metrics as $\delta_{u}$ and $\delta_{v}$. A natural and ubiquitous distance metric in the context of time series is Euclidean distance, and this is what we use to compute the distance between points in each time series. To estimate GMA, we proceed by computing for each $1 \leq i \leq n$, the rank $r_{i}$ of realization $v_{j^{*}}$ in terms of $\delta_{v}$, where $j^{*}$ refers to the index of the closest realization $u_{j}$ to $u_{i}$ in terms of $\delta_{u}$

$$
j^{*}=\arg \min _{j \neq i} \delta_{u}\left(u_{i}, u_{j}\right) .
$$

Let us consider the ranks $\left\{r_{i}\right\}_{i=1}^{n}$ as a random variable $R$; then, the distribution of $R$ will quantify dependence. In fact, if the ranks are broadly distributed, then dependence is small, while skewness of $R$ will mean more dependence. Capturing the skewness can be done in various ways. The adopted approach is very simple and proceeds by calculating the area under the empirical cumulative distribution function (cdf) of $R$. Algorithm 1 describes in detail the steps involved in computing GMA between two time series.

In Algorithm 1, the spread of the ranks is computed to address cases where two or more realizations share the same distance from a third one. Upon completion of the algorithm, $\sum P(R=$ $r)=1$ and, hence, refers to a valid PDF. Therefore, GMA can be mathematically defined as

$$
\mathrm{GMA}=\frac{1}{n-1} \sum_{r=1}^{n-1}(n-r) P(R=r)
$$

where $P(R=r)$ is defined as $P(R=r)=\#\left\{i: r_{i}=r\right\} / n$,

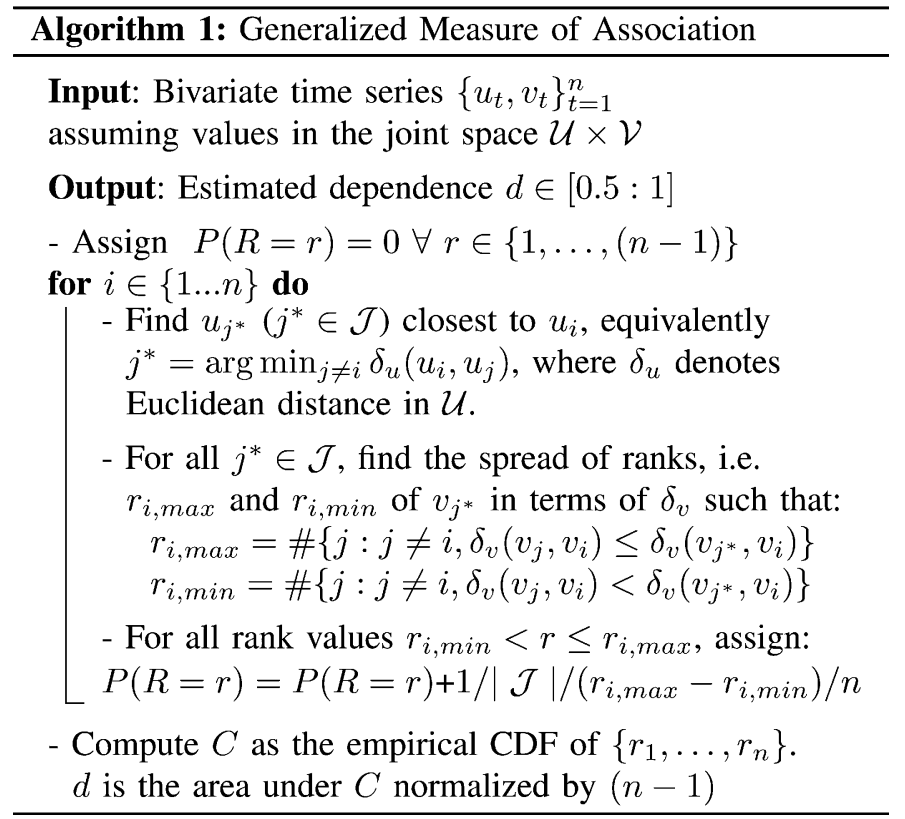

and represents the empirical probability of the rank variable. GMA satisfies the properties of a measure of dependence i.e., it is upper and lower bounded, invariant under a general set of transformations (like rotation and scaling) and can be asymmetric. GMA assumes values between 0.5 and 1 . Being parameterfree, it enjoys a unique computational advantage over other approaches.

\section{Simulations}

As mentioned in the aforementioned section, we use time windows of $114 \mathrm{~ms}$ corresponding to 114 samples given $d=1$ and $F_{s}=1000 \mathrm{~Hz}$. This duration corresponds to two cycles of a sinusoid with frequency $F_{o}$ and satisfies the requirements of having a high time resolution while providing enough samples for dependence computation. When computing correlation and MI, time series were embedded in $\gamma=8$ dimensions to account for the propagation delay among neighboring channels. Tests with absolute correlation were also performed to achieve a better comparison with the other dependence measures since negative correlation values with high magnitude indicate anticorrelation and hence correspond to regions of strong statistical dependence. For the absolute correlation tests, the dependence value was maximized over time lags between 1 and 29 samples. Results were visualized for the whole time duration of $4.2 \mathrm{~s}$, representing a concatenation of 38 time windows.

\section{A. Results}

Each of Figs. 5-8 shows right and left lateral plots of the head for each of the two conditions. GMA was used for Figs. 6 and 7; absolute correlation and MI were used, respectively, for Figs. 5 and 8. Given its asymmetry, GMA plots were comprised of two rows to reflect both directions of the measure. The lower row in each of Figs. 6 and 7 corresponds to dependence values computed from all channel locations to channel 72. 


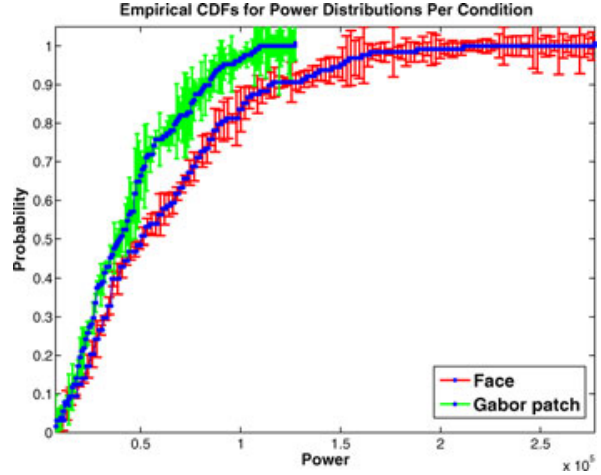

Fig. 4. Power distributions for the two conditions. Error bars represent variability with trials. The KS statistic obtained is 0.2891 with a $p$-value of 0.0003159. The KS statistic is discussed in Section VI-B

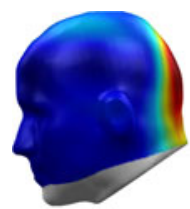

(a) FL - CC

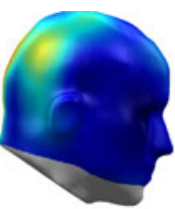

(b) FR - CC

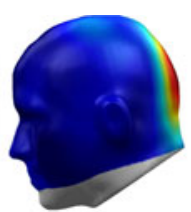

(c) GL - CC

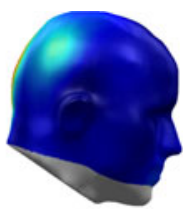

(d) GR - CC
Fig. 5. Using absolute correlation to weight graph connections for channe 72. First two subplots (a) and (b) show interpolated correlation measures over right and left ( $R$ and $L$ ) head surface for the face condition $(F)$ and subsequent subplots (c) and (d) exhibit the same for the Gabor patch condition (G). The active regions are similar for the two cases and variations with time are studied in Fig. 9. A statistical assessment of the discriminatory performance between the two regions is conducted in Section VI-B.
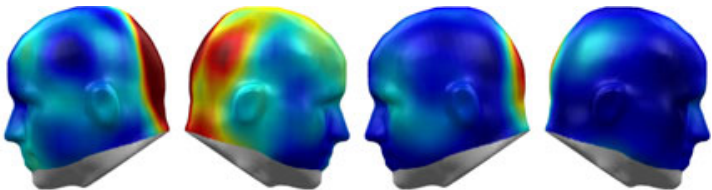

(a) FL - GMA

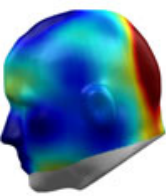

(b) FR - GMAf (a) FL - GMA

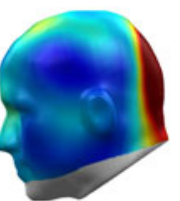

(e) FL - GMA

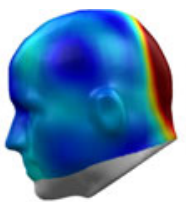

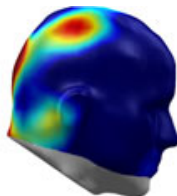

(b) FR - GMA

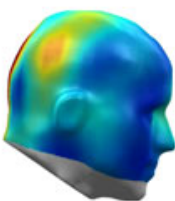

(f) FR - GMA

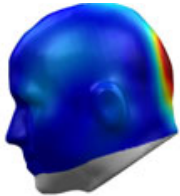

(c) GL - GMA

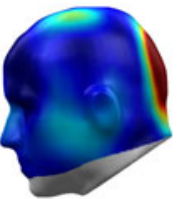

(g) $\mathrm{GL}-\mathrm{GMA}_{b}$

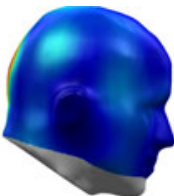

(d) GR - GMA

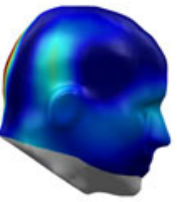

(h) GR - GMA
Fig. 7. Same procedure as in the previous figure applied on the second subject. The similarity in obtained results supports the validity of the analytic procedure across multiple individuals.

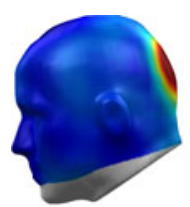

(a) FL - MI

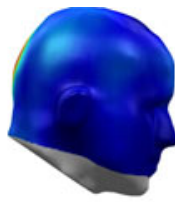

(b) FR - M

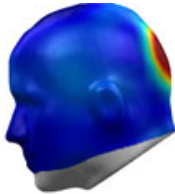

(c) $\mathrm{GL}-\mathrm{M}$

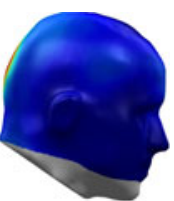

(d) GR - MI
Fig. 8. Same procedure as in Fig. 5 applied for MI. Unlike correlation and GMA, MI shows less discriminability between the two conditions and relatively similar levels of dependence between the two brain hemispheres.

munication between sources in the primary visual cortex and ventral region sources and is consistent with engagement of the fusiform or right occipito-temporal areas. A remarkable observation is that this active region seems to be reinforced with the duration of the presentation. The averaged GMA value in that area shows consistently slight increases with time, noticeable as long as the stimulus is applied, which suggests the reinforcement of communication between sources as time passes.

MI: MI tends to show a more balanced distribution of active regions between the two brain hemispheres as compared to correlation and GMA. Some patterns of right parietal activity can be spotted for the face condition, but their corresponding dependence levels vary across time windows. Similar to the two other dependence measures, a slightly higher level of dependence was observed for that condition.

Dependence Across Time Windows: A subset of electrodes

Fig. 6. Same procedure as in Fig. 5 applied for GMA. The upper row corresponds to forward computations of GMA, i.e., from the selected channel to the others, while the lower row shows the backward computations. More dependence can be seen for the two conditions in the right hemisphere. The noticeable active region for the face condition seems to correspond to medial occipito-temporal structures and might reflect the activity of the fusiform face area. The asymmetry of the measure does not significantly affect the locations of the active regions.

Correlation: Absolute correlation shows consistency in locations of active regions across time and conditions. In addition to visual cortex, superior regions (e.g., superior temporal sulcus) are also implicated.

GMA: Unlike correlation, GMA shows higher dependence for the face condition in the right parietal-temporal-occipital region neighboring $P_{4}$ (Figs. 6 and 7). Although it is too early to be conclusive, this might be explained by an enhanced comcorresponding to the region neighboring electrode location $P_{4}$ [as shown in Fig. 9(a)] was selected to study the variations of our dependence measures across time windows. GMA values exhibit low variability with slight increases as time flows, which might be explained by habituation effects due to presenting the same visual object. On the other hand, absolute correlation and MI show more fluctuations and do not present the same increasing pattern.

\section{B. Statistical Analysis}

To assess quantitatively the dependence maps, we use the twosample Kolmogorov-Smirnov (KS) test, which is a nonparametric test that compares two sample vectors. The KS test tries to 


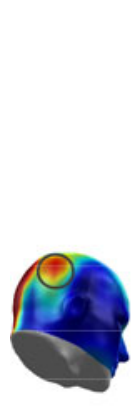

(a)

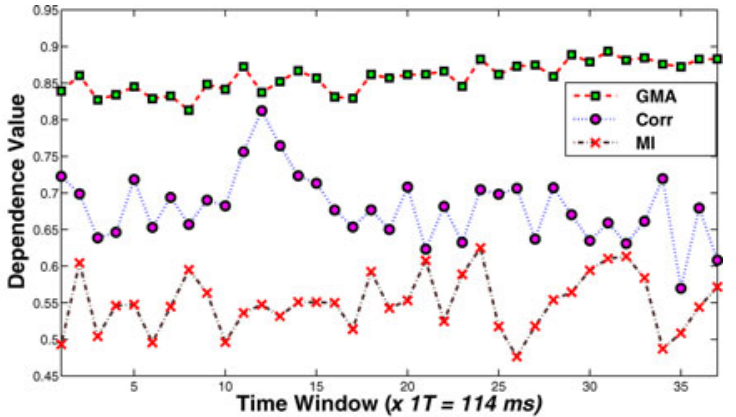

(b)
Fig. 9. (a) Electrode subset selected to track the variations across time windows of dependence values pertaining to the active region. (b) GMA values show more stability across time than absolute correlation and MI.

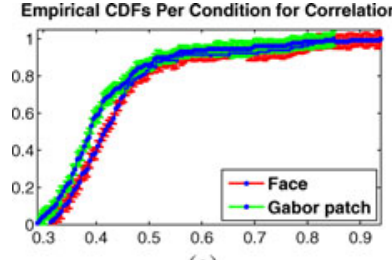

(a)

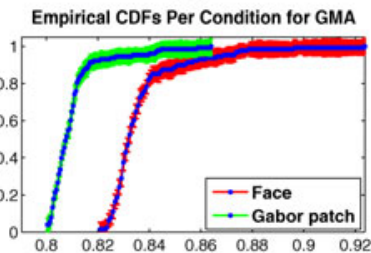

(c)

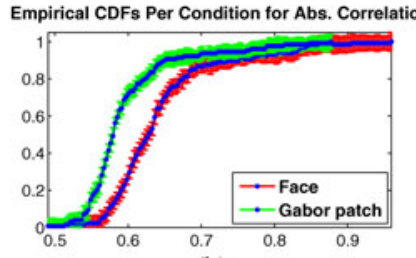

(b)

Empirical CDFs Per Condition for Mutual Information

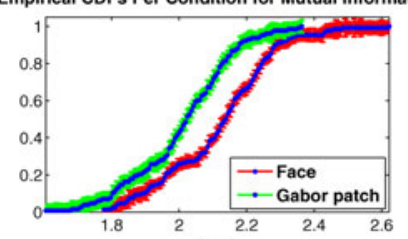

(d)
Fig. 10. Empirical cdfs computed from dependence values across the 129 electrodes and averaged over trials for (a) Pearson's correlation, (b) Absolute Pearson's correlation, (c) MI, and (d) GMA. Time window used is 114 samples or $114 \mathrm{~ms}$. The error bars show the variability with time windows ( 38 windows in this case).

estimate the distance between the empirical distribution functions of the two sets of samples. The null hypothesis is that both samples are drawn from the same distribution. Assuming $\gamma_{1}(x)$ and $\gamma_{2}(x)$ to be the sample vectors, the KS statistic can be calculated as $\mathrm{KS}_{\boldsymbol{\gamma}_{1}, \gamma_{2}}=\max _{x}\left|F_{\boldsymbol{\gamma}_{2}}(x)-F_{\gamma_{1}}(x)\right|$, where $F_{\gamma_{1}}(x)$ and $F_{\gamma_{2}}(x)$ denote the empirical cdfs for the $n$ i.i.d. observations, alternatively $F_{\left\{X_{1}, \ldots, X_{n}\right\}}(x)=\frac{1}{n} \sum_{i=1}^{n} I_{X_{i} \leq x}$, where $I_{k}$ denotes the indicator function. The null hypothesis is rejected at the $\alpha$-level if $\sqrt{\left(n_{1} n_{2}\right) /\left(n_{1}+n_{2}\right)} K S_{\gamma_{1}, \gamma_{2}}>K_{\alpha}$, where $n_{1}$ and $n_{2}$ denote the number of samples from each observation vector and $K$ refers to the Kolmogorov distribution [47].

Fig. 10 shows the empirical distributions per condition for each of the dependence measures considered in this paper across the sensor network and the corresponding value of the KS statistic. Distributions for absolute correlation and MI are more distinct than correlation but show less discriminability than GMA. Looking at a time window of 114 samples, the test statistic obtained for GMA is 0.9125 , compared to 0.3441 and 0.4844 for MI and absolute correlation, respectively, as shown in Fig. 11. All measures performed better than the power classification scheme in Fig. 3. Results were similar when using a longer time

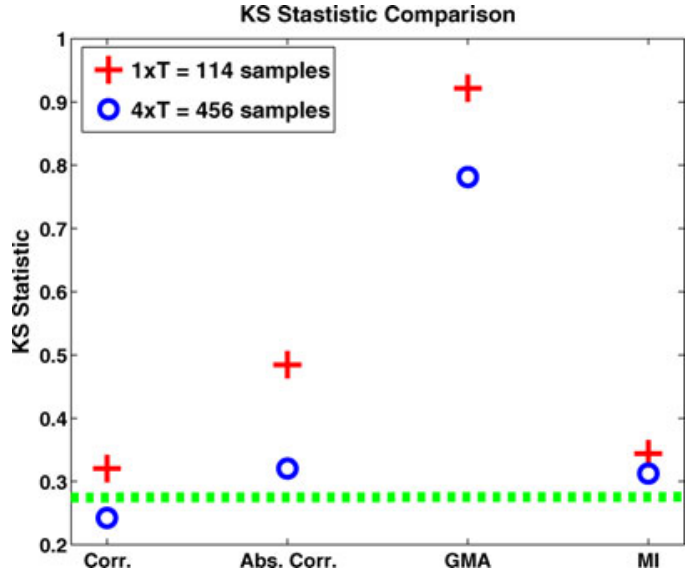

Fig. 11. Test statistic for the two-sample KS test applied for each of the four dependence measures. Points in red were obtained when using time windows of 114 samples and those in blue when using 456 samples. The dotted green line represents the KS statistic obtained using power distributions.

window with a $15 \%$ difference for GMA, when comparing to a smaller time window. The range of values drops for all measures of dependence measures but this comes at the expense of reduced time resolution.

\section{CONCLUSION}

\section{A. Discussion}

This study examined ssVEPs evoked by flickering stimuli showing a human face and Gabor patches. Dependences between channel locations were computed in time windows of $114 \mathrm{~ms}$ for correlation, MI, and a novel measure of dependence, which achieved a better performance in discriminating between the two conditions. Estimates of source locations underlying the ssVEP activity strongly suggest that most scalp-recorded ssVEP signals originate from lower tier visual cortex. This is inline with the findings in [48] and [49] and those in [50] that localize the visual ssVEP in posterior occipital and ventral occipital cortex. Di Russo et al. [48] further suggest that simple ssVEP models consisting of one or two sources in the occipital region of the brain are unable to explain the ssVEPs magnitude and phase, which favors the hypothesis of a distributed source system with enhanced communication between the occipital/parieto-occipital region and sources in ventral regions. Potential sources suggested by this study concur with sources of face-specific responses found by Sams et al. [51].

Although we did not study directional connectivities in this context, the location of active regions witnessed for the face stimulus is consistent with findings suggesting reentrant modulation of early visual cortex, originating from higher tiers and entering lower tiers of visual cortex [3].

\section{B. Further Research}

The data used in this study were based on recordings acquired from two subjects. The next step is to validate the current methodology on extended data obtained from multiple subjects, while studying the intervariability between subjects. It would 
also be interesting to look at the functional connectivities from a perspective involving all channels. A way to do so is to formulate the problem as a complete undirected dependence graph [52] and apply statistical methods to extract the most active edges in the graph. Possible evaluation tools between the two conditions in that case would be graph matching procedures as in [53] and [54]. Using measures of conditional dependence can also be employed to determine directional connectivities. An interesting comparison can be performed to a functional connectivity approach based on coherence analysis [19], [21].

On the other hand, to further elaborate on the obtained results, it would be interesting to infer more information about the nature of communication between the occipito-parietal cortex and the inferotemporal and whether it involves intermediate sources. This can be done by studying the time delay properties of the obtained signals over a set of channels.

\section{REFERENCES}

[1] S. Bressler. (1995). Large-scale cortical networks and cognition, Brain Res. Rev. [Online]. 20(3), pp. 288-304. Available: http://www. sciencedirect.com/science/article/pii/016501739400016I

[2] M. Cole and W. Schneider. (2007). The cognitive control network: Integrated cortical regions with dissociable functions, NeuroImage. [Online]. 37(1), pp. 343-360. Available: http://www.sciencedirect.com/ science/article/pii/S1053811907002820

[3] A. Keil, D. Sabatinelli, M. Ding, P. Lang, N. Ihssen, and S. Heim, "Reentrant projections modulate visual cortex in affective perception: Evidence from Granger causality analysis," Hum. Brain Mapp., vol. 30, pp. 532-540, 2009.

[4] M. Bradley, D. Sabatinelli, P. Lang, J. Fitzsimmons, W. King, and P. Desai, "Activation of the visual cortex in motivated attention," Behav. Neurosci., vol. 117 , pp. 369-380, 2003.

[5] A. Damasio, "Emotion in the perspective of an integrated nervous system," Brain Res. Rev., vol. 26, pp. 83-86, 1998.

[6] M. Davis, "Anatomic and physiologic substrates of emotion in an animal model," Clin. Neurophysiol., vol. 15, pp. 378-387, 1998.

[7] P. Lang, M. Bradley, and B. Cuthbert, "Affect, activation, and action," in Attention and Orienting: Sensory and Motivational Processes. Hillsdale, NJ: Erlbaum, 1997, pp. 97-135.

[8] J. Baizer, R. Desimone, and L. Ungerleider. (1993). Comparison of subcortical connections of inferior temporal and posterior parietal cortex in monkeys, Vis. Neurosci. [Online]. 10(1), pp. 59-72. Available: http://dx.doi.org/10.1017/S0952523800003229

[9] A. Todorov and A. Engell, "The role of the amygdala in implicit evaluation of emotionally neutral faces," Soc. Cogn. Affect. Neurosci., vol. 3, no. 4, pp. 303-312, 2008.

[10] G. Iaria, C. Fox, C. Waite, I. Aharon, and J. Barton, "The contribution of the fusiform gyrus and superior temporal sulcus in processing facial attractiveness: Neuropsychological and neuroimaging evidence," Neurosci., vol. 155, pp. 409-422, 2008.

[11] J. Sergent, S. Ohta, and B. MacDonald, "Functional neuroanatomy of face and object processing: A positron emission tomography study," Brain, vol. 115, pp. 15-36, 1992

[12] J. Anderson, Cognitive Psychology and Its Implications, 6th ed. New York: Worth, 2004.

[13] D. Regan, Human Brain Electrophysiology: Evoked Potentials and Evoked Magnetic Fields in Science and Medicine. New York: American Elsevier, 1989.

[14] A. Keil, S. Moratti, D. Sabatinelli, M. Bradley, and P. Lang, "Additive effects of emotional content and spatial selective attention on electrocortical facilitation," Cereb. Cortex, vol. 15, pp. 1187-1197, 2005.

[15] A. Keil, T. Gruber, M. Mller, S. Moratti, M. Stolarova, M. Bradley, and P. Lang, "Early modulation of visual perception by emotional arousal: Evidence from steady-state visual evoked brain potentials," Cogn. Affect. Behav. Neurosci., vol. 3, pp. 195-206, 2003.

[16] F. Vialatte, M. Maurice, J. Dauwels, and A. Cichockir, "Steady-state visually evoked potentials: Focus on essential paradigms and future perspectives," Phys. Rev. E, vol. 90, no. 4, pp. 418-438, 2010.
[17] S. Moratti, A. Keil, and M. Stolarova, "Motivated attention in emotional picture processing is reflected by activity modulation in cortical attention networks," NeuroImage, vol. 21, pp. 954-964, 2004.

[18] B. Hjorth, "EEG analysis based on time domain properties," Electroencephalogr. Clin. Neurophysiol., vol. 29, no. 3, pp. 306-310, 1970.

[19] R. Srinivasan, W. Winter, J. Ding, and P. Nunez, "EEG and MEG coherence: Measures of functional connectivity at distinct spatial scales of neocortical dynamics," J. Neurosci. Methods, vol. 166, no. 1, pp. 41-52, 2007.

[20] P. Rappelsberger and H. Petsche, "Probability mapping: Power and coherence analyses of cognitive processes," Brain Topogr., vol. 1, no. 1, pp. 46-54, 1988.

[21] S. Makeig, "Using feedforward neural networks to monitor alertness from changes in EEG correlation and coherence," Adv. Neural Inf. Process. Syst., vol. 8, pp. 931-937, 1996.

[22] L. Baccala and K. Sameshima, "Partial directed coherence: A new concept in neural structure determination," Biol. Cybern., vol. 84, pp. 463-474, 2001.

[23] T. Koenig, D. Studer, D. Hubl, L. Melie, and W. Strik, "Brain connectivity at different time-scales measured with EEG," Philos. Trans. B, vol. 360, no. 1, pp. 1015-1027, 2005.

[24] T. Koenig, L. Melie-Garcia, D. Hubl, P. Valdes-Sosa, and T. Dierks, "Multichannel time frequency analysis of spontaneous EEG and fMRI correlates," IFMBE Proc., vol. 360, no. 1, pp. 1015-1027, 2004.

[25] M. Corsi-Cabrera, M. A. Guevara, C. Arce, and J. Ramos, "Inter and intrahemispheric EEG correlation as a function of sleep cycles," Prog. Neuropsychopharmacol. Biol. Psychiatry, vol. 20, pp. 387-405, 1996.

[26] U. Barcaro, F. Denoth, L. Murri, C. Navona, and A. Stefanini, "Changes in the interhemispheric correlation during sleep in normal subjects," Electroencephalogr. Clin. Neurophysiol., vol. 63, pp. 112-118, 1986.

[27] J. Barlow, "Autocorrelation and crosscorrelation analysis," in Handbook of Electroencephalography and Clinical Neurophysiology, Frequency and Correlation Analysis. Amsterdam, The Netherlands: Elsevier, 1973, pp. 79-96.

[28] M. Guevaraa and M. Corsi-Cabrera, "EEG coherence or EEG correlation?" Int. J. Psychophysiol., vol. 23, no. 3, pp. 145-153, 1966.

[29] S. Na, S. Jin, S. Kim, and B. Ham, "EEG in schizophrenic patients: Mutual information analysis," Clin. Neurophysiol., vol. 113, no. 12, pp. 19541960, 2002

[30] C. Honey, R. Kotter, M. Breakspear, and O. Sporns, "Network structure of cerebral cortex shapes functional connectivity on multiple time scales," Proc. Natl. Acad. Sci. U.S.A., vol. 104, pp. 10240-10245, 2007.

[31] J. Jeong, J. Gore, and B. Peterson, "Mutual information analysis of the EEG in patients with Alzheimer's disease," Clin. Neurophysiol., vol. 112, no. 5 , pp. 827-835, 2001.

[32] E. G. Inc. (2007). Geodesic sensor net technical manual. [Online.] pp. 29-30. Available: http://www.egi.com.

[33] H. Strasburger, W. Scheidler, and I. Rentschler, "Amplitude and phase characteristics of the steady-state visual evoked potential," Appl. Opt. vol. 27, no. 6, pp. 1069-1088, 1988.

[34] E. Phelps, S. Ling, and M. Carrasco, "Emotion facilitates perception and potentiates the perceptual benefits of attention," Psychol. Sci., vol. 17, no. 4, pp. 292-299, 2006

[35] P. Bex, S. Solomon, and S. Dakin, "Contrast sensitivity in natural scenes depends on edge as well as spatial frequency structure," J. Vis., vol. 9, no. 10, pp. 1-19, 2009.

[36] M. Wieser and A. Keil, "Temporal trade-off effects in sustained attention: Dynamics in visual cortex predict the target detection performance during distraction," J. Neurosci., vol. 31, no. 21, pp. 7784-7790, 2011.

[37] H. Wieringa, MEG, EEG and the integration With Magnetic Resonance Images, The Netherlands: University of Twente, 1993.

[38] C. Nicholson and J. Freeman, "Theory of current source-density analysis and determination of conductivity tensor for anuran cerebellum," $J$. Neurophysiol., vol. 38, no. 2, pp. 356-368, 1975.

[39] C. Tenke and J. Kayser. (2005). Reference-free quantification of EEG spectra: Combining current source density (CSD) and frequency principal components analysis (fPCA), Clin. Neurophysiol. [Online]. 116(12), pp. 2826-2846. Available: http://www.sciencedirect. $\mathrm{com} / \mathrm{science} /$ article/pii/S138824570500324X

[40] M. Junghofer, T. Elbert, P. Leiderer, P. Berg, and B. Rockstroh, "Mapping EEG-potentials on the surface of the brain: A strategy for uncovering cortical sources," Brain Topogr., vol. 9, no. 9, pp. 203-217, 1997.

[41] M. Codispoti, V. Ferrari, A. D. Cesarei, and R. Cardinale, "Implicit and explicit categorization of natural scenes," Prog. Brain Res., vol. 156, pp. 53-65, 2006. 
[42] M. Codispoti, V. Ferrari, M. Junghofer, and H. Schup, "The categorization of natural scenes: Brain attention networks revealed by dense sensor ERPs," NeuroImage, vol. 32, pp. 583-559, 2006.

[43] B. Fadlallah, S. Seth, A. Keil, and J. Principe, "Robust EEG preprocessing for dependence-based condition discrimination," in Proc. 33rd Annu. IEEE Eng. Med. Biol. Soc., Sep. 2011, pp. 1407-1410.

[44] A. Keil, J. Smith, B. Wangelin, D. Sabatinelli, M. Bradley, and P. Lang, "Electrocortical and electrodermal responses covary as a function of emotional arousal: A single-trial analysis," Psychophysiology, vol. 45, no. 4, pp. 516-523, 2008.

[45] A. Kraskov, H. Stögbauer, and P. Grassberger. (2004). Estimating mutual information, Phys. Rev. E. [Online]. 69(6), p. 066138-1-066138-16. Available: http://link.aps.org/doi/10.1103/PhysRevE.69.066138

[46] S. Seth, A. Brockmeier, J. Choi, M. Semework, J. Francis, and J. Principe, "Evaluating dependence in spike train metric spaces," IEEE Int. Joint Conf. Neural Netw., pp. 2645-2652, 2011.

[47] G. Marsaglia, W. Tsang, and J. Wang, "Evaluating Kolmogorov's distribution," J. Stat. Softw., vol. 8, pp. 1-4, 2003.

[48] F. Di Russo, S. Pitzalis, T. Aprile, G. Spitoni, F. Patria, A. Stella, D. Spinelli, and S. Hillyard, "Spatiotemporal analysis of the cortical sources of the steady-state visual evoked potential," Hum. Brain Mapp., vol. 28, pp. 323-333, 2007.

[49] M. Wieser and A. Keil, "Temporal trade-off effects in sustained attention: Dynamics in visual cortex predict the target detection performance during distraction," J. Neurosci., vol. 31, pp. 7784-7779, 2011.

[50] M. Muller, W. Teder, and S. Hillyard, "Magnetoencephalographic recording of steady-state visual evoked cortical activity," Brain Topogr., vol. 9, pp. $163-168,1997$.

[51] M. Sams, J. Hietanen, R. Hari, R. Ilmoniemi, and O. Lounasmaa, "Facespecific responses from the human inferior occipito-temporal cortex," Neurosci., vol. 77, no. 1, pp. 49-55, 1997.

[52] B. Fadlallah, S. Seth, A. Keil, and J. Principe, "Analyzing dependence structure of the human brain in response to visual stimuli," presented at the 37th Annu. IEEE Int. Conf. Acoust., Speech, Signal Process., Kyoto, Japan, 2012.

[53] D. Conte, F. Foggia, C. Sansone, and M. Vento, "Thirty years of graph matching in pattern recognition," Int. J. Pattern. Recognit., vol. 18, no. 3 , pp. 265-298, 2004.

[54] E. Zegura, K. Calvert, and M. Donahoo, "A quantitative comparison of graph-based models for internet topology," IEEE/ACM Trans. Netw., vol. 5, no. 6, pp. 770-783, Dec. 1997.

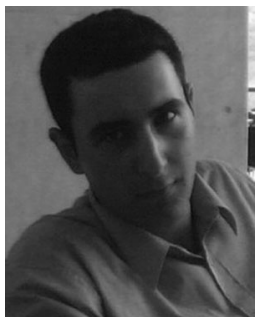

Bilal Fadlallah (M'09) received the B.E. degree in computer and communications engineering and the M.E. degree in electrical and computer engineering from the American University of Beirut, New York, NY, in 2006 and 2008, respectively. He is currently working toward the Ph.D. degree in electrical and computer engineering in the Computational NeuroEngineering Laboratory, University of Florida, Gainesville.

His research interests and industry experience include the fields of signal processing, machine learning, computational neuroscience, and software engineering.

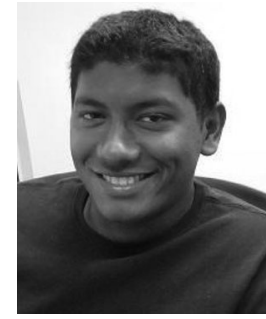

Sohan Seth (M'11) received the Ph.D. degree from the Department of Electrical and Computer Engineering, the University of Florida, Gainesville, in 2011.

$\mathrm{He}$ is currently a Postdoctoral Researcher at the Helsinki Institute for Information Technology, Helsinki, Finland. He is also with the Department of Information and Computer Science, Aalto University, Aalto, Finland. His research interests include the fields of machine learning, data mining, and computational neuroscience.

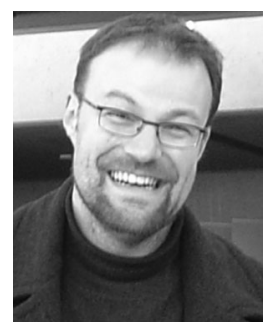

Andreas Keil received the Ph.D. degree in psychology from the University of Konstanz, in 2000.

He is currently an Associate Professor of psychology at the University of Florida, Gainesville, where he is also a member of the NIMH Center for the Study of Emotion and Attention. His research focuses on attention and perception in the human visual system. $\mathrm{He}$ is particularly interested in how the visual brain changes - over the life span and also as a consequence of short-term intervention- to optimize processing of information that is relevant for the individual.

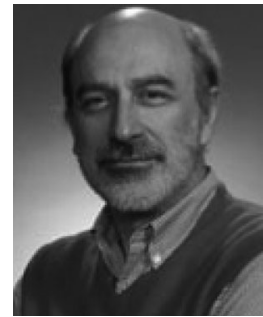

José Príncipe (F'00) received the Ph.D. degree in electrical engineering from the University of Florida, Gainesville, in 1977.

He is currently a Distinguished Professor of electrical and computer engineering with the University of Florida. He is also a BellSouth Professor and the Founding Director of the Computational Neuro-Engineering Laboratory, University of Florida. His current research interests are focused on advanced signal processing and machine learning, plications of cognitive systems. brain-machine interfaces, and the modeling and ap- 